\title{
Internal multiple suppression by adaptive Marchenko redatuming
}

Joost van der Neut, Kees Wapenaar, Jan Thorbecke (Delft University of Technology) and Ivan Vasconcelos (Schlumberger Gould Research)

\section{SUMMARY}

Recently, a novel iterative scheme was proposed to retrieve Green's functions in an unknown medium from its single-sided reflection response and an estimate of the propagation velocity. In Marchenko imaging, these Green's functions are used for seismic imaging with complete wavefields, including internal multiple reflections. In this way, common artifacts from these internal reflections are avoided and illumination of the subsurface can potentially be improved. However, Marchenko imaging requires accurate input data, with correct amplitudes, a deconvolved source signature, without free-surface multiples and source / receiver ghosts. Hence, a significant amount of preprocessing is required, which should be done accurately. To relax these requirements, we propose a scheme to remove artifacts due to internal multiples from inverse-extrapolated wavefields, by adaptively subtracting an estimate of these artifacts that is constructed with the Marchenko equation.

\section{INTRODUCTION}

Internal multiple reflections can pose severe challenges for seismic imaging algorithms, that commonly assume that a wavefield reflects only once in the subsurface. Hence, a variety of methods have been developed to remove internal multiple reflections from seismic data (Weglein et al., 1997; Berkhout and Verschuur, 2005). Recently, a novel methodology was proposed to estimate Green's functions from single-sided reflection data and an estimate of the propagation velocity (Wapenaar et al., 2014a). Since internal multiple reflections can be predicted by this scheme, they can be included in the imaging process to suppress multiple-related artifacts in a data-driven manner (Wapenaar et al., 2014b). The iterative scheme that constitutes the core of Marchenko imaging involves multiple crosscorrelations with the recorded data. Here, it is assumed that the source wavelet, ghosts and free-surface multiples have been removed from the data before entering the scheme and that amplitudes are accurately recorded. If these assumptions are not or poorly fulfilled, the retrieved events are likely to contain errors. In this paper, we take a closer look at the process in which internal multiples are constructed by the scheme and we propose an adaptive filter to improve robustness in cases where the current requirements on the input data are not met. This leads to a new adaptive scheme for the suppression of imaging artifacts caused by internal multiple scattering.

\section{MARCHENKO REDATUMING}

Wapenaar et al. (2014b) derived two Green's function representations that we can discretize as

$$
\begin{aligned}
& \mathbf{g}^{-}=\mathbf{R f}_{d}^{+}+\mathbf{R f}_{m}^{+}-\mathbf{f}^{-}, \\
& \mathbf{g}^{+}=-\mathbf{R Z \mathbf { Z }} \mathbf{f}^{-}+\mathbf{Z \mathbf { f } _ { d } ^ { + }}+\mathbf{Z \mathbf { f } _ { m } ^ { + }} .
\end{aligned}
$$

In these representations, $\mathbf{g}^{-}$and $\mathbf{g}^{+}$are the up- and downgoing Green's functions, respectively, with sources at the surface and receivers at a desired focal point in the subsurface. Further, we find the focusing function $\mathbf{f}=\mathbf{f}_{d}^{+}+\mathbf{f}_{m}^{+}+\mathbf{f}^{-}$, which has been decomposed in three terms: a downgoing direct field $\mathbf{f}_{d}^{+}$, a downgoing coda $\mathbf{f}_{m}^{+}$and an upgoing part $\mathbf{f}^{-}$. All vectors are expressed as column vectors with concatenated traces in the time-space domain. Matrix $\mathbf{R}$ applies multidimensional convolution with the reflection response (e.g. the observed data at the surface), whereas matrix $\mathbf{Z}$ applies time reversal to any vector by rearranging its elements. To arrive at the coupled Marchenko equations, we design a muting matrix M, that removes the direct arrival and all events after this arrival from the gathers. Because of causality, $\mathbf{M g}^{ \pm}=\mathbf{0}$. It can be shown that $\mathbf{M Z f}_{d}^{+}=\mathbf{0}, \mathbf{M Z f}_{m}^{+}=\mathbf{Z f}_{m}^{+}$and $\mathbf{M f}^{-}=\mathbf{f}^{-}$(Wapenaar et al., 2014a). Although these equations have been proven to hold for media with smoothly curved interfaces, their limitations are still to be investigated in more complex media. Here we assume they hold, such that applying $\mathbf{M}$ to equations 1 and 2 (after re-arranging) yields

$$
\begin{gathered}
\mathbf{f}^{-}=\mathbf{M R f}_{d}^{+}+\mathbf{M R f}_{m}^{+}, \\
\mathbf{Z f}_{m}^{+}=\text {MRZf }^{-} .
\end{gathered}
$$

In this scheme, $\mathbf{f}_{d}^{+}$is the initial focusing function. It can be obtained by time-reversal of the direct wave between the surface locations and the focal points that can be obtained from a background velocity model. The key idea of Marchenko imaging is to estimate $\mathbf{f}^{-}$and $\mathbf{f}_{m}^{+}$by iteratively updating equations 3 and 4, followed by Green's function retrieval with equations 1 and 2. Once the Green's functions are known to various locations at a specified focusing level, the seismic wavefield can be redatumed to this level by multidimensional deconvolution of $\mathbf{g}^{-}$with $\mathbf{g}^{+}$. By repeating this exercise at each depth level in the subsurface and evaluating the result at zero time lag, an image can be created with substantially suppressed artifacts (Slob et al., 2014; Broggini et al., 2014). Alternatively, the data can be redatumed to a specified level (for instance below a complex near-subsurface or salt body) and conventional imaging can proceed below this level (Wapenaar et al., 2014b).

\section{INTERFEROMETRIC INTERPRETATION}

Each update of the focusing function with equation 3 or 4 can be interpreted as a multidimensional crosscorrelation with the 


\section{Adaptive Marchenko redatuming}

reflection response. Inspired by the literature on seismic interferometry, Van der Neut et al. (2014) interpreted each crosscorrelation by subtracting traveltimes along common raypaths at the stationary points of the underlying integrals (Schuster, 2009). In this way, we can obtain a clear understanding of how particular events are retrieved by the scheme. We illustrate the interferometric interpretation for a $2 \mathrm{D}$ synthetic subsalt imaging experiment, that will be evaluated later on in this abstract. In Figure 1a, we show the synthetic model that is used to generate reflection data at the surface. In Figure 1b, we show a smooth version of the model, which we use to generate the direct field. The initial focusing function is obtained by timereversing this field. The solid red line depicts the acquisition array at the surface, where 161 sources and 161 receivers are deployed. The white line is a focusing level and the magenta dot represents a single focal point at this level. Our aim is to image the faulted structure between the two salt bodies. If we do so by conventional imaging, strong internal multiples from the upper salt body cause artifacts, as we show later. Hence, we want to redatum the data to the focusing level by Marchenko redatuming, thereby removing the effects from internal multiple reflections, and generate a local image from the redatumed data.

The initial focusing function acts as an inverse wavefield extrapolator to the reflection response. In Figure 2a, we illustrate how primary upgoing events in $\mathbf{g}^{-}$are constructed by applying the reflection response $\mathbf{R}$ to $\mathbf{f}_{d}^{+}$, which is the first term in the right-hand side of equation 1 . Internal multiples in the upgoing field are retrieved as well, as demonstrated in Figure $2 b$. However, we also observe artifacts, as illustrated in Figure 2c. The traveltime of this artifact is obtained by adding the traveltimes along the (positive) red raypaths and subtracting the traveltimes along the (negative) green raypaths, all evaluated at the stationary points of the underlying integrals (Van der Neut et al., 2014).

The iterative scheme will update the focusing function by $\mathbf{f}_{m 1}^{+}$, such that artifacts as in Figure $2 \mathrm{c}$ are canceled when the second term in equation 1 is added to the first term. To illustrate this, we focus on the first estimates of $\mathbf{f}_{1}^{-}$and $\mathbf{f}_{m 1}^{+}$that are obtained by the initial updates of equations 3 and 4 (where subscript 1 indicates that we consider the first update only). From equation 3 , we find

$$
\mathbf{f}_{1}^{-}=\mathbf{M R f}_{d}^{+} .
$$

In Figure 3a, we show a particular event that is created by this action. Once more, the traveltime of this event is found by subtracting the traveltime along the green raypath from the traveltime along the red raypath. We proceed the scheme by timereversing $\mathbf{f}_{1}^{-}$and convolving it with the reflection response, following equation 4 . This brings us the first-order estimate of $\mathbf{Z \mathbf { f } _ { m } ^ { + }}$ :

$$
\mathbf{Z f}_{m 1}^{+}=\mathbf{M R Z f}_{1}^{-}=\mathbf{M R Z M R f}_{d}^{+} .
$$

In Figure $3 b$, we show a particular event that is created by this action. Once again, its traveltime is obtained by subtracting the traveltimes along the green raypaths from the traveltimes
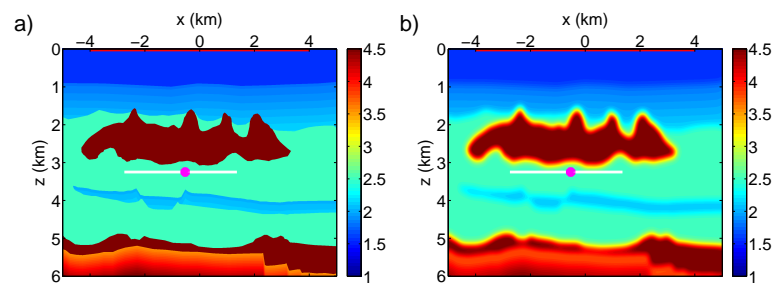

Figure 1: a) Synthetic velocity model (in $\mathrm{km} / \mathrm{s}$ ) in which the reflection response at the surface is computed to construct $\mathbf{R}$. b) A smooth background model, in which the direct arrival is computed to construct $\mathbf{f}_{d}^{+}$.

a)
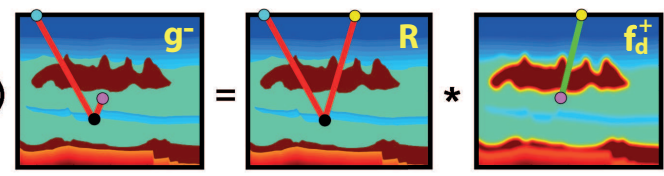

b)
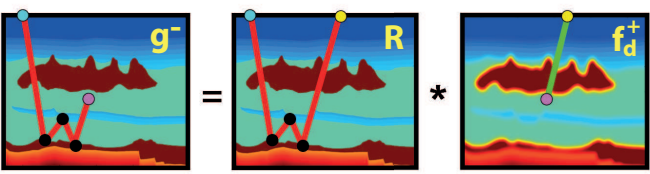

c)
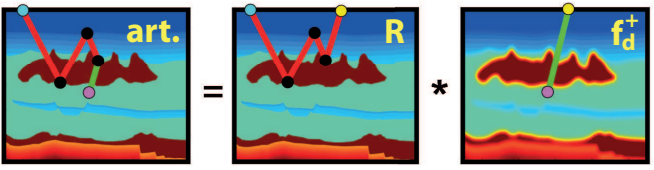

Figure 2: Several events that emerge when $\mathbf{R}$ (middle panels) is applied to $\mathbf{f}_{d}^{+}$(right panels): a) primary reflections in the upgoing field, b) internal multiples in the upgoing field and c) artifacts. Red rays have positive traveltime and green rays have negative traveltimes. These rays are visualized at the stationary points of the underlying integrals.

along the red raypaths. When $\mathbf{Z f _ { m 1 } ^ { + }}$ is time-reversed (note that $\mathbf{f}_{m 1}^{+}=\mathbf{Z Z f}_{m 1}^{+}$) and convolved with the reflection response, as in equation 1 , we retrieve events with similar kinematics as the artifacts that were presented in Figure 2c, as demonstrated in Figure 3c. However, since the events predicted by $\mathbf{f}_{d}^{+}$and $\mathbf{f}_{m}^{+}$have opposite polarity (Wapenaar et al., 2013), they cancel each other. Although complete cancellation requires updating the scheme through iteration, the amplitudes of the first update tend to be already quite accurate for first-order internal multiples.

\section{ADAPTIVE SUBTRACTION}

In this section, we will approximate $\mathbf{f}^{-}$and $\mathbf{f}_{m}^{-}$by their estimates $\mathbf{f}_{1}^{-}$and $\mathbf{f}_{m 1}^{-}$, as in equations 5 and 6 . The upgoing Green's function will be constructed with equation 1 . Since $\mathbf{M \mathbf { f } ^ { - }}=\mathbf{f}^{-}$, the last term in equation 1 has no contribution after the direct arrival time. We make use of this fact, by applying the matrix $(\mathbf{I}-\mathbf{M})$ to equation 1, where $\mathbf{I}$ is an identity matrix, yielding

$$
\mathbf{g}^{-} \approx \mathbf{g}_{0}^{-}+\alpha * \mathbf{g}_{\Delta 1}^{-}
$$




\section{Adaptive Marchenko redatuming}

Here, we have defined

$$
\mathbf{g}_{0}^{-}=(\mathbf{I}-\mathbf{M}) \mathbf{R f}_{d}^{+},
$$

and

$$
\mathbf{g}_{\Delta 1}^{-}=(\mathbf{I}-\mathbf{M}) \mathbf{R f}_{m 1}^{+}=(\mathbf{I}-\mathbf{M}) \mathbf{R Z M R Z M R f}_{d}^{+} .
$$

Further, we have introduced an adaptive filter $\alpha$ in equation 6 that can be used to match the amplitudes of $\mathbf{g}_{0}^{-}$and $\mathbf{g}_{\Delta 1}^{-}$. Inspired by Surface-Related Multiple Elimination (where a similar filter is used to subtract predicted free-surface multiples from recorded data), $\alpha$ is a short convolutional filter, which is chosen such that $\left|\mathbf{g}^{-}\right|_{2}$ is minimized, where subscript 2 represents the $l_{2}$-norm (Verschuur and Berkhout, 1997). This filter can compensate for inaccurate amplitudes, but also the source wavelet (in case of no or inaccurate deconvolution) and (remnant) source / receiver ghosts could be accounted for.

\section{RESULTS}

In this section, we apply the proposed adaptive procedure to 2D synthetic data that was computed in the model of Figure 1a. Our initial goal is to retrieve the upgoing field at the focal point, indicated by the magenta dot in the figure. For reference, we have also computed this field by finite difference modeling and wavefield decomposition, see Figure $4 \mathrm{a}$. To compute $\mathbf{g}_{0}^{-}$, we apply matrix $\mathbf{R}$ to the initial focusing function (see equation 8). The result is shown in Figure 4b, where the red curve defines the arrival time of the direct wave. The field $\mathbf{g}_{0}^{-}$is constructed by muting all samples above this curve (as done by the filter $(\mathbf{I}-\mathbf{M})$ in equation 8$)$. We compute $\mathbf{f}_{m 1}^{+}$with equation 6 and apply $\mathbf{R}$ to the result, see Figure 5a. The field $\mathbf{g}_{\Delta 1}^{-}$ is constructed by muting all information above the red curve (see equation 9). In Figure 5b, we show the result after adaptively subtracting $\mathbf{g}_{\Delta 1}^{-}$from $\mathbf{g}_{0}^{-}$. A few things can be noticed. In the blue box, we observe an event in Figure $4 \mathrm{~b}$ that does not belong to the upgoing Green's function in Figure 4a. Hence, this is an artifact that should be removed by higher-order iterations of the scheme. In Figure 5a, we see the same event with opposite polarity. Note that this artifact is effectively removed in Figure 5b. In the yellow box, an event is clearly visible in Figure $4 \mathrm{a}$, but hiding below artifacts in Figure $4 \mathrm{~b}$. Since these artifacts are well predicted in Figure 5a (with opposite polarity), they have been effectively subtracted in Figure 5b. The underlying event is now clearly visible. We retrieve the upgoing Green's functions across the focusing level that is indicated by the white line in Figure 1a. Next, we migrate the retrieved upgoing fields in a target area below the focusing level, using the smooth velocity model. In Figure 6a, we show the true velocity model of the target area. In Figure $6 b$, we show the image that is obtained when $\mathbf{g}_{0}^{-}$is migrated (representing a conventional image). Besides the fault structure that we are after, we can observe many artifacts caused by internal multiples in the overburden. In Figure 7a, we show a migration image of $-\alpha * \mathbf{g}_{\Delta 1}^{-}$, where we reversed the polarity for illustrative purposes. Note that the predicted artifacts align well with a)
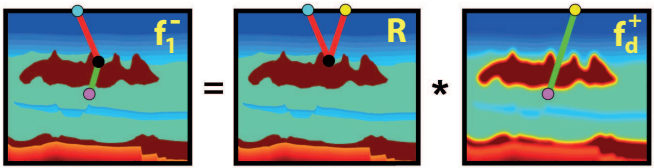

b)
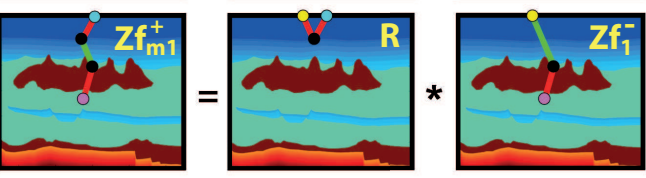

c)
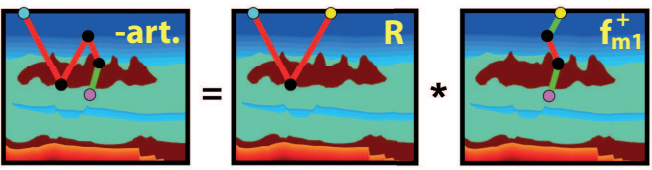

Figure 3: The retrieval of an event that cancels the artifact in Figure 2c. a) The initial focusing function $\mathbf{f}_{d}^{+}$(right panel) is convolved with $\mathbf{R}$ (middle panel) to produce an event in $\mathbf{f}_{1}^{-}$ (left panel) through equation 5. b) This event is time-reversed (right panel) and convolved with $\mathbf{R}$ (middle panel) to produce an event in $\mathbf{Z \mathbf { f } _ { m 1 } ^ { + }}$ (left panel) through equation 6. c) This event is time-reversed (right panel) and convolved with $\mathbf{R}$ (middle panel) through equation 1 . The result is an event with similar kinematics but reversed polarity as the event in Figure 2c.

the artifacts in the previous figure. Hence, when both figures are subtracted, the artifacts are eliminated, see Figure $7 b$.

\section{DISCUSSION AND CONCLUSION}

The image in Figure $7 \mathrm{~b}$ is not perfect yet. The illumination is incomplete and reflectors have been slightly mispositioned. This can be attributed to the finite aperture and the velocity smoothening that was applied to construct the initial focusing function. We should also notice that not all internal multiples have been eliminated by the followed procedure. The retrieved upgoing field should be interpreted as the response at the focusing level to a downgoing field that includes internal multiples. To remove these internal multiples as well, redatuming can be applied by multidimensional deconvolution of the retrieved upgoing field with the retrieved downgoing field (Wapenaar et al., 2014b). It was shown by Van der Neut et al. (2013) that also this step can be implemented by adaptive subtraction, when developed as a Neumann series. In conclusion, we have developed a methodology for the adaptive subtraction of internal multiples, based on the first updates of the iterative scheme that undergirds Marchenko redatuming. In the future, higher-order updates may be included as well.

\section{ACKNOWLEDGEMENTS}

The research of J. van der Neut was sponsored by the Technology Foundation STW, applied science foundation of NWO (project 13078). We thank Carlos Almagro Vidal (Delft University of Technology) for creating and sharing the 2D synthetic model that was used in this abstract. 


\section{Adaptive Marchenko redatuming}

a)

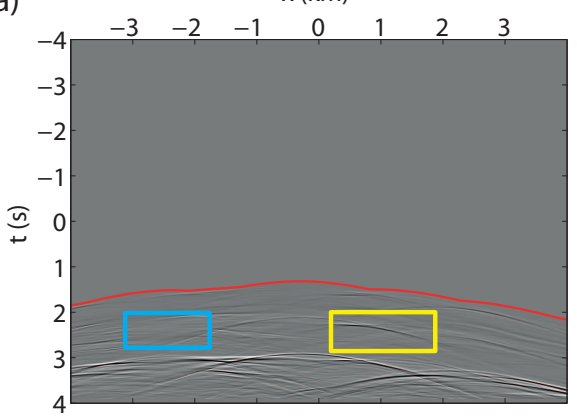

b)

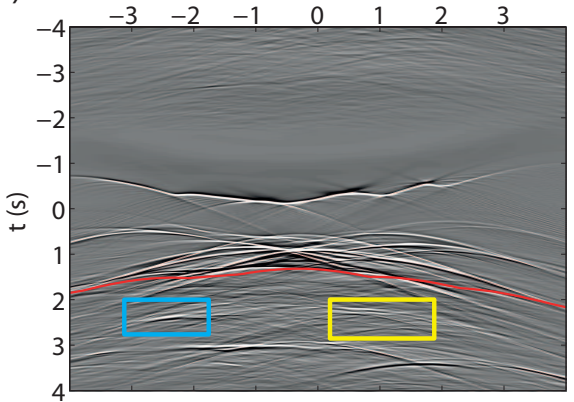

Figure 4: a) Desired upgoing field $\mathbf{g}^{-}$, obtained by direct modeling. b) Result of $\mathbf{R f}_{d}^{+}$. The red curve defines the direct arrival time. Matrix $\mathbf{M}$ removes all information below this curve, whereas matrix $(\mathbf{I}-\mathbf{M})$ removes the information above the curve. Hence, all data below the red curve defines $\mathbf{g}_{0}^{-}$.

a)

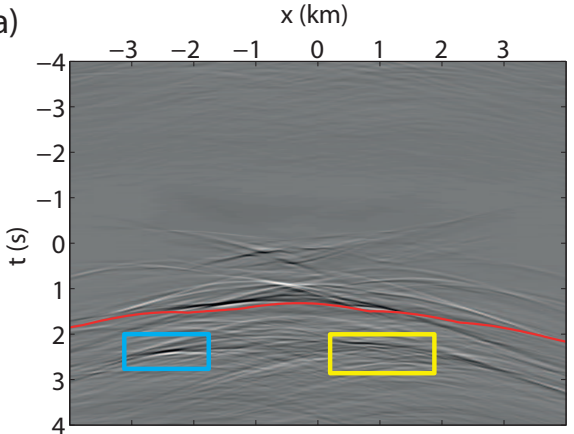

b)

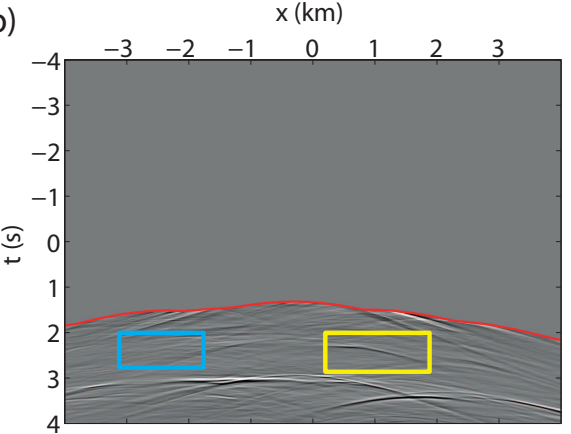

Figure 5: a) Result of $\mathbf{R f}_{m 1}^{+}$. All data below the red curve defines $\mathbf{g}_{\Delta 1}^{-}$. b) Result of adaptive subtraction of $\mathbf{g}_{\Delta 1}^{-}$from $\mathbf{g}_{0}^{-}$.
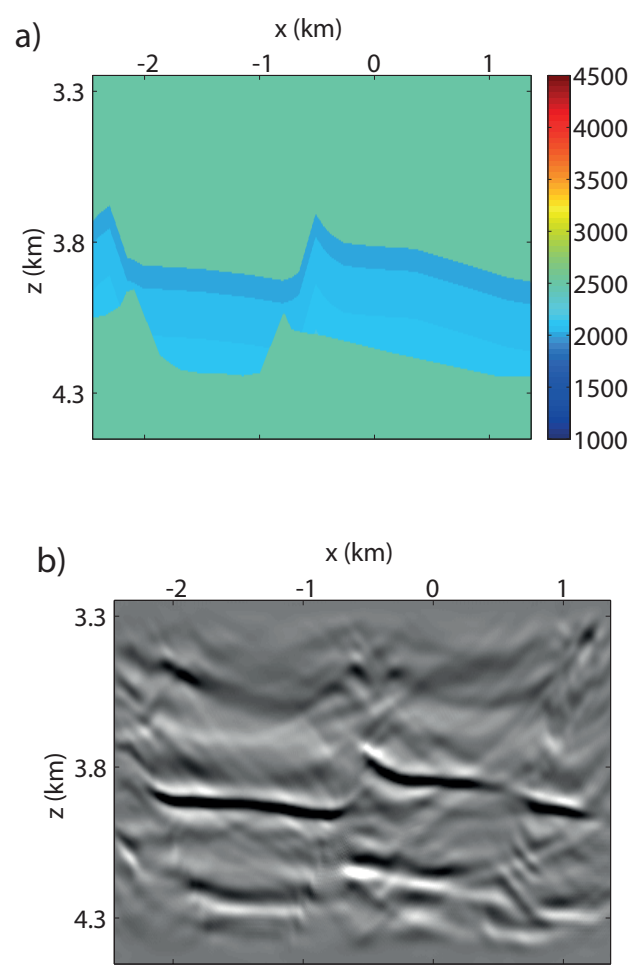

Figure 6: a) Model of the target area below the white focusing level in Figure 1a. b) Image of the target area by migration of $\mathbf{g}_{0}^{-}$.

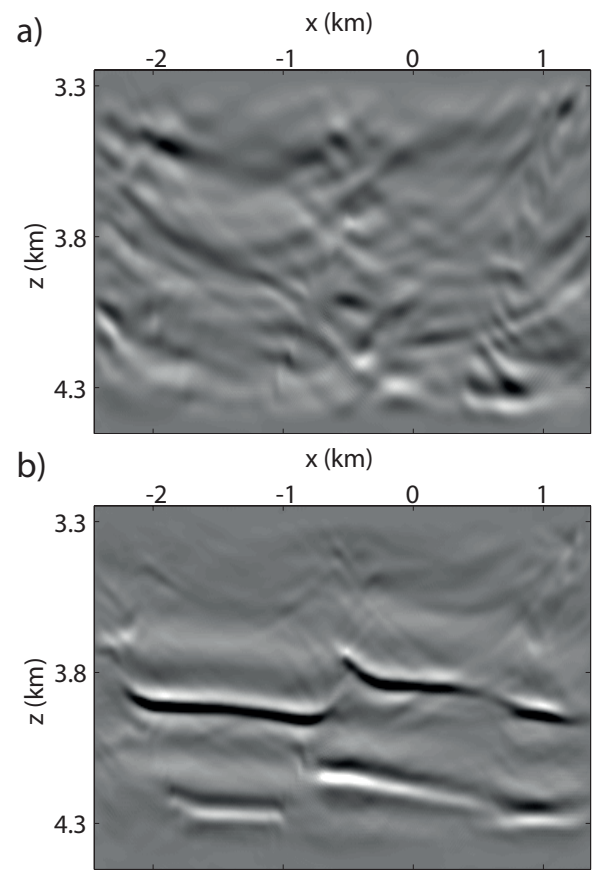

Figure 7: a) Image of $-\alpha \mathbf{g}_{\Delta 1}^{-}$in the target area. b) Image of the target area, after $-\alpha \mathbf{g}_{\Delta 1}^{-}$has been subtracted from $\mathbf{g}_{0}^{-}$. 


\section{Adaptive Marchenko redatuming}

\section{REFERENCES}

Berkhout, A. J. and D. J. Verschuur, 2005, Removal of internal multiples with the common focus-point (cfp) approach: part 1 explanation of the theory: Geophysics, 70, V45-V60.

Broggini, F., K. Wapenaar, J. van der Neut, and R. Snieder, 2014, Data-driven green's function retrieval and application to imaging with multidimensional deconvolution: Journal of Geophysical Research - Solid Earth, 119, 425-444.

Schuster, G. T., 2009, Seismic interferometry: Cambridge University Press.

Slob, E., K. Wapenaar, F. Broggini, and R. Snieder, 2014, Seismic reflector imaging using internal multiples with marchenko-type equations: Geophysics, 79, S63-S76.

Van der Neut, J., E. Slob, K. Wapenaar, J. Thorbecke, R. Snieder, and F. Broggini, 2013, Interferometric redatuming of autofocused primaries and internal multiples: 83rd Annual Meeting, SEG, Expanded Abstracts.

Van der Neut, J., I. Vasconcelos, and K. Wapenaar, 2014, An interferometric interpretation of marchenko redatuming: 76th EAGE Conference and Exhibition, Extended Abstracts.

Verschuur, D. J. and A. J. Berkhout, 1997, Estimation of multiple scattering by iterative inversion, part ii: practical aspects and examples: Geophysics, 62, 1595-1611.

Wapenaar, K., F. Broggini, E. Slob, and R. Snieder, 2013, Three-dimensional single-sided marchenko inverse scattering, data-driven focusing, green's function retrieval and their mutual relations: Physical Review Letters, 110, 084301.

Wapenaar, K., J. Thorbecke, J. van der Neut, F. Broggini, E. Slob, and R. Snieder, 2014a, Green's function retrieval from reflection data, in absence of a receiver at the virtual source position: Journal of the Acoustical Society of America, 135, (accepted).

, 2014b, Marchenko redatuming: Geophysics, 79, (accepted).

Weglein, A. B., F. A. Gasparotto, P. M. Carvalho, and R. H. Stolt, 1997, An inverse-scattering series method for attenuating multiples in seismic reflection data: Geophysics, 12, 1975-1989. 